\title{
Mme de Duras, Ourika. Édouard. Olivier ou le Secret
}

\section{Silvia Lorusso}

\section{(Q) OpenEdition}

\section{Journals}

\section{Edizione digitale}

URL: http://journals.openedition.org/studifrancesi/8959

DOI: 10.4000/studifrancesi.8959

ISSN: 2421-5856

\section{Editore}

Rosenberg \& Sellier

\section{Edizione cartacea}

Data di pubblicazione: 1 octobre 2008

Paginazione: 469-470

ISSN: 0039-2944

\section{Notizia bibliografica digitale}

Silvia Lorusso, «Mme de Duras, Ourika. Édouard. Olivier ou le Secret», Studi Francesi [Online], 155 (LII I

II) | 2008, online dal 30 novembre 2015, consultato il 08 janvier 2021. URL: http://

journals.openedition.org/studifrancesi/8959; DOI: https://doi.org/10.4000/studifrancesi.8959

\section{Questo documento è stato generato automaticamente il 8 janvier 2021.}

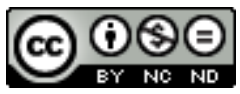

Studi Francesi è distribuita con Licenza Creative Commons Attribuzione - Non commerciale - Non opere derivate 4.0 Internazionale. 


\title{
Mme de Duras, Ourika. Édouard. Olivier ou le Secret
}

\author{
Silvia Lorusso
}

\section{NOTIZIA}

MME DE DURAS, Ourika. Édouard. Olivier ou le Secret, préface de Marc Fumaroli, édition de Marie-Bénédicte DIETHELM, Paris, Gallimard, 2007 («Folio classique»), pp. 402.

1 Questo libro concede per la prima volta al lettore di trovare riuniti, in un unico volume, i tre romanzi di Madame de Duras. Già i testi di Ourika e Édouard presentano importanti variazioni rispetto a edizioni correnti: per esempio, in Édouard, l'exergue tratto da Tasso non si trova né nell'edizione curata da Claudine Hermann per «Mercure de France», né in quella «Bouquins» 1996, che non riporta neppure la Conclusion del romanzo. Ancora più importanti sono le differenze relative a Olivier.

2 In questa nuova stampa, infatti, diversamente che da quella curata da Denise Virieux, che per prima pubblicò il romanzo presso Corti nel 1971 a partire da un abbozzo autografo, è riportato il testo definitivo del manoscritto autografo d'olivier ou le Secret, conservato negli archivi di famiglia della duchessa de Duras e trascritto da Madame Diethelm, con lievissime accortezze da lei dichiarate nella «Note sur l'établissement des textes». Dell'esistenza di questo manoscritto si poteva solo supporre, poiché SainteBeuve cita nei suoi Portraits, a proposito di Olivier, l'immagine del «mur de cristal» (presente anche in Armance e trasformata da Stendhal in «mur de diamant») che non si trova nel testo pubblicato da Virieux.

Questa nuova edizione si rivela dunque ben più lunga della precedente, arricchita da brani o dettagli fino a oggi inediti. Così l'episodio della morte di M. de Nangis presenta uno sviluppo molto più articolato (lettera XVI della I parte); come anche il racconto della gita all'isola di Wight (lettera IX della III parte), dove per altro è presente un nuovo personaggio, il barone de T., e quello di sua moglie, Henriette de D., riceve maggiori dettagli (lettera VIII sempre della III parte). Inoltre, come ci dice Madame 
Diethelm, il ritrovamento di questo manoscritto permette anche di chiarire diversi dubbi su alcune definitive scelte da parte di Madame de Duras (quale potrebbe essere l'incertezza tra due nomi per un personaggio). Per giungere infine alla novità più notevole che è data da una nota inedita della Conclusion del romanzo, in cui la narratrice insinua che Olivier si sarebbe ucciso perché sapeva di essere il fratello di Louise. Congettura (per altro "demeurée sans preuve», come sempre la narratrice ci dice), ben diversa da quella lasciata intuire nelle letture private da salotto, in cui Madame de Duras aveva fatto supporre, quale causa della tragedia, l'impotenza dell'eroe «spiegazione quest'ultima sicuramente più diffusa all'epoca, come testimonia l'Armance di Stendhal. Tale nota inedita apporta una novità consistente rispetto al romanzo prima conosciuto, perché confonde il lettore; ma ha anche il merito di abbandonarlo «à une méditation sur les méandres insolubles et insondables du cœur humain», come conclude la curatrice del libro.

4 Al guadagno apportato ai testi dei tre romanzi di Claire de Duras, il presente volume aggiunge un dettagliatissimo e ricco apparato critico. In apertura la «préface» di Marc Fumaroli inquadra i romanzi nella loro epoca, rivelandone l'importanza per più grandi scrittori come Stendhal o Balzac. E rintraccia episodi reali che sarebbero all'origine delle tre storie narrate da Madame de Duras. Dalla romanzesca e sfortunata vita della duchessa colpita più volte nei sentimenti più forti (il padre condannato a morte per aver rifiutato di firmare a favore della morte del re; il matrimonio sfortunato; la separazione dalle figlie; il legame profondo e doloroso con Chateaubriand) viene fuori il ritratto di una donna brillante, sofferente, melanconica, a tratti disperata, in perenne attesa dell'oggetto d'amore: «épouse déçue, fille d'un martyr, mère privée de sa fille adorée, amie ardente d'un "frère" à éclipses». E ciascuno dei suoi romanzi descrive questa disperazione e l'impossibilità della felicità, in quanto: «À supposer même que soient levés les obstacles occasionnels qui interdisent le bonheur aux héros de ces trois romans, il resterait que le désir de bonheur, [...] porte en lui-même le principe d'une désillusion mélancolique qui conduit nécessairement soit à Dieu, soit à la mort». Secondo Fumaroli, gli ostacoli che si frappongono tra gli amanti sarebbero dunque poco importanti in quanto tali. Diverrebbero quasi degli strumenti funzionali a una concezione del desiderio che non può trovare appagamento nella realtà, ma soltanto nella morte o in Dio. Tuttavia questa inappagabilità amorosa, variante femminile del mal du siècle, mi pare non possa prescindere dal punto di vista romanzesco dall'importanza attribuita agli ostacoli. Il romanzesco si concentra nella loro elaborazione; sono loro che consentono di costruire trame e di disegnare caratteri.

Alla «Préface» di Fumaroli segue l'«Introduction» di Marie-Bénédicte Diethelm che si propone di segnalare il valore dell'opera di Madame de Duras (la curatrice preferisce Édouard) e il suo posto nella letteratura non solo della sua epoca. Parte dalla constatazione dell'enorme successo di pubblico che Ourika ebbe «settemila esemplari, contro i mille di Armance, per altro non tutti venduti; inoltre la moda di bluse all'Ourika e cappelli all'Ourika» a cui si affiancò quello di critica. Quindi Madame Diethelm riporta con grande precisione tutte le vicissitudini editoriali dei tre romanzi, dalle loro letture private fino alla successiva decisione di darli alle stampe per i primi due. Per quanto riguarda Olivier, invece, viene illustrata la vicenda relativa alla pubblicazione da parte di Latouche di un Olivier apparso anonimo e sconfessato da Madame de Duras che lascerà il suo inedito. 
6 Infine la curatrice racconta gli avvenimenti che sarebbero alla base di queste tre storie che sono così collegate alla vita privata della duchessa: «L'essentiel, en effet, n'est pas dans ces thèmes, mais dans ce que ces personnages nous disent de la souffrance d'une femme supérieurement intelligente, torturée par la conviction "qu'elle n'avait pas ce qu'il faut pour être aimée". À cette âme malheureuse, il est suggéré de "calmer son imagination assombrie en écrivant"». Mme de Duras è delusa dal suo matrimonio e poi anche dal legame con Chateaubriand: in fondo i suoi romanzi racconterebbero tutti, in uno stile, preciso, elegante, che nulla concede all'artificio e alla moda, la storia di un uomo che rifiuta l'amore di una donna. Questo fondo di amarezza psicologica e amorale, giustamente individuato dalla Diethelm all'origine dei romanzi, non mi pare però che possa rendere non pertinente la loro costruzione romanzesca che ha un debito col codice narrativo all'epoca imperante. Se il senso delle storie è sempre lo stesso, le diverse modalità narrative che lo procurano (gli ostacoli, i ritardi) risultano altrettanto importanti.

7 Dopo il testo dei tre romanzi, il volume presenta una dettagliata «Chronologie» della vita della duchessa (1777-1828), seguita da un'altra cronologia che rende conto di tutti i suoi scritti, compresi quelli mai pubblicati. Quindi vi è una «Note sur l'établissement des textes», di cui ho reso conto avanti; un «Document» con un estratto dal diario inedito di Madame de Duras (a proposito di Ourika); una ricca «Bibliographie sélective». Infine le fondamentali «Notes» ai tre romanzi, sia di carattere editoriale, che di commento. 